fainter component $B$ has an axial period of rotation equal to its orbital period of revolution with respect to the brighter component $A$. In addition, the hemisphere of $B$ exposed to the radiation from $A$ is brighter than the other hemisphere; this was the first time that this 'reflexion effect' had been observed. When Stebbins took up the study of Algol ten years later, using the more accurate photo-electric photometer, not only were the general features of the binary as determined with the selenium cell confirmed, but also another effect arising from the slightly ellipsoidal shape of the components was detected.

Algol, however, was one of the exceptions to Eddington's mass-luminosity relation on the assump. tion that the mass of $A$ was 0.37 that of the sun and the mass of $B$ half that of $A$. Later, when McLaughlin and Rossiter, using a new method, applied it to the solution of the binary problem, it was found that $A$ was five times as massive as $B$, and $A$ then satisfies the mass-luminosity requirements, thus vindicating the accuracy and importance of Stebbins's work.

At the total solar eclipses of 1918, 1925 and 1937, Stebbins measured the integrated light of the corona with his photo-electric apparatus, and concluded that there is little evidence of any noticeable variation of the total light of the corona during the sunspot period. Another interesting result was that the light of the corona, photo-electrically, is about one-half that of the full moon. From the absence of evidence of the Rayleigh scattering, it was inferred that the continuous and Fraunhofer spectrum can be produced by electrons or large dust particles, but not by atoms, molecules and particles of wave-length dimensions. Finally, over a large range of wave-lengths the intensity distributions for the sun and corona are the same- a fact which must be taken into consideration by all who formulate any general theory of the corona.

It is impossible to refer to all the branches of Stebbins's work, and it will be sufficient to mention one or two others of outstanding importance. In 1930, when he visited Lick Observatory, he turned his attention to an extensive observational programme on stars (starting with $B$ stars), globular clusters and extragalactic nebulæ. This programme was so comprehensive and so important that it is well worth reading Prof. Smart's address to realize the implications of some of the results obtained. It may be mentioned that the evidence obtained from the globular clusters led to a revision not only of their individual distances but also of the diameter of the galaxy, which was shown to be considerably less than that of Shapley's original estimate and is now believed to be about 100,000 light-years or less. This reduction in the dimensions of our galaxy, combined with Stebbins's work on the Andromeda nebula with the photo-electric observations of its outlying regions, which showed it to be considerably larger than was originally believed, has led to the conclusion that there is little difference in the overall dimensions of the two systems.

In 1947 Stebbins attempted to locate the central nucleus of the galaxy. Using the 60-inch telescope at Mt. Wilson, the galvanometer deflexions for the infra-red filter $(10,300 \mathrm{~A}$.) and also for a red filter (7,190 A.) were noted in narrow zones, with the telescope stationary, in the neighbourhood of $326^{\circ}$ galactic longitude. The observations extended over several degrees in longitude and latitude, and the maxima of the infra-red readings appeared to indicate a bulge. An analysis of the space-reddening showed that the light of the bulge, extending over $8^{\circ}$ in longitude and $4-5^{\circ}$ in latitude, when corrected for absorption, is comparable with the light of an equivalent section of the Andromeda nebula. The position, outline and total light of the bulge suggest that something is being detected near the galactic centre, but Stebbins makes no greater claim than this.

Prof. Stebbins's work is well summed up at the end of Prof. Smart's address : "In surveying our Medallist's work over the long period of nearly fifty years one cannot but be impressed by his devotion to one great department of astronomy which he in great measure has made his own, by his observational skill, his attention to detail and his critical analysis of the new and unexpected phenomena which his observations have brought to light".

\section{INSTITUTION OF GAS ENGINEERS}

\section{AUTUMN RESEARCH MEETING}

$T$ HE sixteenth autumn research meeting of the Institution of Gas Engineers was held in London during November 28-29, 1950, with Mr. F. M. Birks as president.

Like other public services, the gas industry has problems of peak load, if of less intensity. Normally they are met by the use of carburetted water gas, a product which makes the combined use of gasified coke and mineral oil. H. Stanier and J. B. McKean described a new process based on the gasification of mineral oil, blended with steam and heated in contact with a suitable catalyst. Under suitable conditions the product is a gas of composition and character similar to normal town's gas, and can be used for supplementing the latter at peak periods. From economic considerations this process might provide an alternative to gas manufactured from coal.

At the present time, the sulphur available from home sources is obtained by the purification of coal gas. The normal methods of recovery, although efficient, have certain practical difficulties, and any improved means might be an important contribution to the national economy. E. Johnson described an attempt to recover sulphur of high purity from the spent oxide of gas purification practice using a fluidized technique and solvent extraction. A sulphur product of 95 per cent purity is obtained, and the extracted oxide has a reactivity to hydrogen sulphide not greatly different from that of the original material. The work has shown that a semi-continuous process for the extraction of sulphur from spent oxide in a fluidized bed by means of solvents is possible, and no fundamental difficulties are likely to be encountered in making the process fully continuous. A large-scale fluidized process was proposed for further investigations.

The importance of combustion research in gas utilization was emphasized by J, E. Garside in a review of the field of relevant research. Flame propagation, flame stability, mechanism of combustion reactions, heat and energy release, heat and energy transfer, and ignition of gas-air mixtures were all briefly dealt with, and the extent of present-day knowledge indicated. Some of the major questions facing gas utilization in the industry at the present time were stated, and an attempt was made to answer them by explaining the findings of current researches in Great Britain and correlating those findings with the results of similar projects overseas. 